\title{
Successful Management of a 29-Day-Old Infant with Severe Hyperlipidemia from a Novel Homozygous Variant of GPIHBP1 Gene
}

Shu Liu ( shmicy@163.com )

Guangdong Women and Children Hospital

\section{Zhiqing Wang}

Southern Medical University Nanfang Hospital

\section{Xianhua Zheng}

Guangdong Women and Children Hospital

\section{Ye Zhang}

Guangdong Women and Children Hospital

\section{Sisi Wei}

Guangdong Women and Children Hospital

\section{Haimei OuYang}

Guangdong Women and Children Hospital

Jinqun Liang

Guangdong Women and Children Hospital

Nuan Chen

Guangdong Women and Children Hospital

\section{Weihong Zeng}

Guangdong Women and Children Hospital

Jianhui Jiang

Guangdong Women and Children Hospital

\section{Short report}

Keywords: Severe hyperlipidemia; Whole exome sequencing; GPIHBP1 gene, Novel variant; Therapy

Posted Date: October 8th, 2021

DOI: https://doi.org/10.21203/rs.3.rs-955186/v1

License: (1) (1) This work is licensed under a Creative Commons Attribution 4.0 International License.

Read Full License 


\section{Abstract}

Background: Severe hyperlipidemia is characterized by markedly elevated blood triglyceride levels and severe early-onset cardiovascular diseases, pancreatitis, pancreatic necrosis or persistent multiple organ failure if left untreated. It is a rare autosomal recessive metabolic disorder originated from the variants of lipoprotein lipase gene, and previous studies have demonstrated that most cases with severe hyperlipidemia are closely related to the variants of some key genes for lipolysis, such as $L P L, A P O C 2$, $A P O A 5, \angle M F 1$ and GPIHBP1. Meanwhile, other unidentified causes also exist and are equally worthy of attention.

Methods: The 29-day-old infant was diagnosed with severe hyperlipidemia, registering a plasma triglyceride level as high as $25.46 \mathrm{mmol} / \mathrm{L}$. Whole exome sequencing was conducted to explore the possible pathogenic gene variants for this patient.

Results: The infant was put on a low-fat diet combined with pharmacological therapy, which was successful in restraining the level of serum triglyceride and total cholesterol to a low to medium range during the follow-ups. The patient was found to be a rare novel homozygous duplication variantc.45_48dupGCGG (Pro17Alafs*22) in GPIHBP1 gene-leading to a frameshift which failed to form the canonical termination codon TGA. The mutant messenger RNA should presumably produce a peptide consisting of 16 amino acids at the N-terminus, with 21 novel amino acids on the heels of the wild-type protein.

Conclusions: Our study expands on the spectrum of GPIHBP1 variants and contributes to a more comprehensive understanding of the genetic diagnosis, genetic counseling, and multimodality therapy of families with severe hyperlipidemia. Our experience gained in this study is also contributory to a deeper insight into severe hyperlipidemia and highlights the importance of molecular genetic tests.

\section{Background}

Severe hyperlipidemia, with a typical feature of high concentration of lipidemia in fasting state, is primarily attributed to a genetic defect in intravascular lipolysis. Urgent clinical intervention is always required, especially for serious cases in that some severe complications, otherwise, may follow, such as pancreatitis, which contributes to $5 \%-6 \%$ of the overall mortality, as well as pancreatic necrosis or persistent multiple organ failure that have been proved to be associated with the highest mortality rate [1]. Amongst all the subtypes of severe hyperlipidemia, monogenic forms are the ones linked to the defects in metabolism of triglyceride (TG)-rich lipoproteins, namely lipolytic cascade. These defects may be caused by variants in no less than five different genes, predominantly (about 95\%) inherited ones in both alleles of the lipoprotein lipase gene, which, aliased as $L P L$ [2], is responsible for encoding of the enzyme lipoprotein lipase (LPL; OMIM \#238600). Previous studies have shown that the defects directly related to $L P L$ gene variants are also relevant to the majority of severe hyperlipidemia cases [3-6]. The rest $5 \%$ are the results of variants in other genes involving in LPL functioning, including APOC2 (encoding 
apolipoprotein CIl, activator of LPL; OMIM \#207750) [7, 8], APOA5 (encoding apolipoprotein AV, activator of LPL; OMIM \#144650) [9, 10], LMF1 (encoding lipase maturation factor 1, a tissue factor triggering the secretion of functional LPL and hepatic lipase; OMIM \#611761) [11, 12], and GPIHBP1 (encoding glycosylphosphatidylinositol-anchored highdensity lipoprotein-binding protein 1, the molecular platform by which LPL is able to interact with TG-rich lipoproteins, apolipoprotein CII, and apolipoprotein AV on the endothelial surface of capillaries; OMIM \#612757) $[13,14]$. Meanwhile, there are also other variants to be identified [3], and all these variants may result in LPL malfunction.

GPIHBP1, categorized as one of the lymphocyte antigen 6 (Ly6) family, is a capillary endothelial cell protein consisting of 184 aa. Being a critical protein for LPL transportation from the subendothelial spaces to capillary lumen, GPIHBP1 can promote lipolysis by working as the main site to bind LPL on endothelial surface [15]. So, variants of GPIHBP1 may disturb lipolysis process and thus result in severe hyperlipidemia. However, although previous studies so far have offered some leads to the lipolytic mechanism of GPIHBP1 and disclosed a handful of case reports concerning GPIHBP1 variants, our understanding on GPIHBP1-deficient diseases is still to be deepened. In this study, we described the clinical features, genetic analysis and our hands-on experience of successful pharmacological management of a 29-day-old baby with abnormally elevated plasma TG $(25.46 \mathrm{mmol} / \mathrm{L})$, who was subsequently identified as a rare novel homozygous duplication variant in GPIHBP1 gene as the cause of her severe hyperlipidemia.

\section{Materials And Methods Clinical information}

The patient was a 29-day-old Chinese female infant with a chief complaint of "irritability, fever, vomiting and convulsion for 2 days", who was enrolled in our study after consultation in the pediatric clinic of our hospital. The patient responded poorly to the treatment in previous visits to her local hospitals due to the limited health care resources there, making her condition worsening with the time. Therefore, she was referred to our hospital and finally placed in the pediatric intensive care unit. After aggressive treatment, the infant was stablized in vital signs, with physical examinations showing normal muscle strength, muscle tension and physiological reflexes. Unexpected discovery arose from milky blood sample (lactescent plasma) and the results of lipid testing (in fasting state), which revealed abnormally elevated TG (25.46 mmol/L; normal range, 0.46 to $2.28 \mathrm{mmol} / \mathrm{L}$ ) as well as abnormal cholesterol metabolismtotal cholesterol (TC, $14.53 \mathrm{mmol} / \mathrm{L}$; normal range, 3.12 to $5.68 \mathrm{mmol} / \mathrm{L}$ ), high-density lipoprotein cholesterol (HDL-C, $1.12 \mathrm{mmol} / \mathrm{L}$; normal range, 0.91 to $2.18 \mathrm{mmol} / \mathrm{L}$ ), and low-density lipoprotein cholesterol (LDL-C, $2.01 \mathrm{mmol} / \mathrm{L}$; normal range, 1.28 to $3.41 \mathrm{mmol} / \mathrm{L}$ ). Based on these findings, hyperlipidemia was suspected despite the fact that the patient's parents were healthy with normal levels of serum lipid and lipoprotein, and the family history indicated neither hyperlipidemia nor pancreatitis. Therefore, aiming to identify the possible pathogenic gene variants for this infant, we performed this study using genetic analysis tools, with 100 Chinese healthy subjects recruited as the control group. 


\section{Genetic Analysis}

Enlightened by the abnormal clinical findings, hereditary hyperlipidemia was taken into the pediatricians' consideration for further confirmation using whole exome sequencing (WES, Illumina, San Diego, CA, USA). Details of the experiments, mutation frequency investigation, variant verification, and assessment of the conservation of amino acid residues were displayed in the Supplementary document (shown in Supplementary document-genetic analysis). The pathogenicity of the sequence variants was interpreted according to the American College of Medical Genetics and Genomics/Association for Molecular Pathology (ACMG/AMP) guidelines [16, 17].

The study was approved by the Ethics Committee of Guangdong Women and Children Hospital. Informed consent was given by the patient's parents, along with the authorization to the photographs, clinical studies and laboratory tests, as well as the publication of our work. All the procedures carried out in our study were strictly in accordance with the Declaration of Helsinki.

\section{Results}

\section{Clinical features and therapy procedures of this patient}

The infant is the first child of the non-consanguineous parents, with a full-term birth weight of $2600 \mathrm{~g}$. After the patient was hospitalized, a physical examination was performed in the wake of history collection. On examination, the height of this exclusively breastfed infant was $52 \mathrm{~cm}$ (22.6th percentile), and the weight $3.7 \mathrm{~kg}$ (16.9th percentile). Appearance of malnutrition, poor response to external stimuli, and somnolence were noticed, but without any special face or limb deformity, or dysmyotonia observed. Typical manifestations for dyslipidemia such as xanthomas, abdominal pain or hepatosplenomegaly were also not spotted. The first therapeutic intervention for the infant was oral therapy free of long chain fatty acids, supplemented with high protein, high carbohydrate, and medium chain triglycerides (MCT), which lasted for 5 days but failed to control hyperlipidemia. Due to the potential risk of acute pancreatitis associated with high levels of plasma TG and the signs of hypoproteinemia, enteral feeding was discontinued at the day when the baby was one month and five days old and substituted by multimodality therapy including fasting, nasogastric drainage, total parenteral nutrition (TPN), and antiinfection treatment. After 7 days (one month and twelve days old), plasma TG level decreased significantly to $1.50 \mathrm{mmol} / \mathrm{L}$ and TC to $9.66 \mathrm{mmol} / \mathrm{L}$. Whereas an evident rise in plasma TG up to 3.77 $\mathrm{mmol} / \mathrm{L}$ was, again, detected after switching to breastfeeding for 5 days (one month and seventeen days old). So the infant was put on a low-lipid formula diet, mainly STOLLE milk powder (Stolle Milk Biologics International Incorporated, New Zealand), a kind of formula low in fat and high in protein with proper amount of water-soluble vitamins added. As a result, the plasma TG level declined promptly, ending up at $2.92 \mathrm{mmol} / \mathrm{L}$ when the infant was discharged from the hospital (two month and three days old).

Considering the evidence of lipometabolic disturbance and presumed diagnosis of severe hereditary hyperlipidemia, the infant, after discharged, continued to be kept on a low-fat and high-carbohydrate 
protocol with proper amount of protein diet and supplementation of L-carnitine, coenzyme Q10 as well as water-soluble vitamins (B1, B2, B6, B12, C and folic acid) during her treatment at home. Individualized dosage was given for her medication protocol and ajusted accordingly based on her clinical manifestation, the level of blood lipid, and other nutritional indicators (shown in Supplementary table 1). Surveillance of her medical condition was also performed to avoid potential complications. Meanwhile, the infant continued to take examinations in our outpatient clinic, irregularly, on and off, because of poor compliance. In the follow-ups, the baby was monitored by serum TG, TC, HDL-C, LDL-C, apoprotein A1 (APO-A1), apoprotein B (APO-B), and glucose every three to six months (shown in Supplementary table 2). Besides, evaluation of her physical development was conducted at a three-month interval. The results revealed that the serum TG and TC kept fluctuating from low to medium levels (Fig. 1), and in the last follow-up when the baby was nine months old, she was $8.6 \mathrm{~kg}$ (42.2th centile) in weight and $72 \mathrm{~cm}$ (56.1 th centile) in height, with a head circumference of $44.5 \mathrm{~cm}$ (56.9th centile), indicating a good health and normal development. Likewise, laboratory tests also showed dramatic improvement as compared with her first visit, reflecting a sound developmental condition of the patient as well as the beneficial outcomes of this treatment protocol.

\section{Genetic Findings}

Durinbg hospitalization, the WES for this infant revealed the presence of a homozygous duplication variant-c.45_48dupGCGG(Pro17Alafs*22)-in exons 1 of GPIHBP1 gene (Fig. 2A, 2B). However, being heterozygote carriers of the variant, the parents did not show any symptoms of the disorder. This variant was computationally predicted to be deleterious and morbigenous by CADD, PROVEAN, and Mutation Taster. Results of bioinformatic analysis also strongly suggested it a disease-causing variant. Moreover, this variant was not detected in any of the 100 Chinese healthy subjects in the control group, and also had not been disclosed by any public databases as mentioned above, thus ruling out the possibility of a polymorphism and suggesting its novel and rare occurrence. Ultimately, the variant was identified as a pathogenic factor according to the Sherloc/ACMG criteria: it was not recorded in any of the existing population databases, and was assumed to be closely associated with a highly conserved amino acid, and also, to be disease-causing based on in silico algorithms used for pathogenicity prediction [17]. In this case, the c.45_48dupGCGG duplication variant in exon 1 led to the substitution of the remaining 168 C-terminal amino acid residues with 21 mutant ones (Fig. 2D), and the Ly- 6 antigen/uPA receptor-like domain of this protein was also affected, likely to cause a direct impairment on the enzymatic activity of GPIHBP1. Meanwhile, the functional significance of the mutated amino acid is evident considering its high evolutionary conservation from mammals to invertebrates (Fig. 2C). The outcomes from bioinformatic analysis strongly support that the novel homozygous variant c.45_48dupGCGG(Pro17Alafs*22) is with a disease-causing nature.

As far as we know, this is, in Chinese population, the first report of baby patient with hyperlipidemia due to GPIHBP1 variant, and also the first case with the variant of c.45_48dupGCGG(Pro17Alafs ${ }^{\star 22}$ ) in GPIHBP1 gene that was disclosed worldwide among patients with severe hyperlipidemia. 


\section{Discussion}

In the present study, we delineated a 29-day-old female baby with incidentally found severe hyperlipidemia originated from a novel and homozygous GPIHBP1 duplication variant, who was successfully managed by low-fat diet combined with pharmacological therapy.

As is known that elevated plasma triglyceride may, as an independent risk factor, lead to cardiovascular diseases (CVDs). LPL plays a critical role in lipid metabolism and energy balancing by hydrolyzing the triglyceride in blood circulation. Although it is parenchymal cells that synthesize and secret LPL, it somehow acts on the luminal capillary endothelium, so the mechanism of LPL migrating into the luminal capillary has long been among the list of relevant research [18]. According to previous studies, hyperlipidemia is very likely to be triggered by gene variants which consequently cause LPL dysfunction [19-21]. It has been known that multiple factors can interact with LPL, positively or negatively, thus exerting an influence on TG lipolysis. Among them is a recently identified factor GPIHBP1, which is considered indispensible in transportation of LPL to the luminal capillary endothelium as well as in the establishment of the platform for TG hydrolysis [22]. Currently, a large amount of evidence supports that GPIHBP1 functions in triglyceride-rich lipoprotein (TRL) metabolism of human in unique and diverse ways [23-25]. Recent studies have shown that LPL mislocalization as a result of GPIHBP1 deficiency may cause severe hyperlipidemia $[13,26]$. Therefore, the importance of GPIHBP1 in lipolysis is being recognized more widely with more relevant research published.

It was reported that loka et al. first identified GPIHBP1 as a protein with an HDL binding capability [15]. Nevertheless, disputes about its HDL-binding ability has been existed ever since until its function in lipolysis became unumbiguous due to the finding of significantly elevated plasma triglyceride levels in GPIHBP1 knockout mice [27]. Moreover, GPIHBP1 was proved to be able to bind not only LPL, but also chylomicrons, and the activity of LPL after heparin processing was compromised in knockout mice. Thereafter, looking into its mechanism of how to handle triglycerides has always been one of the highlights of such studies. It has already been known that LPL migrating into luminal capillary is a prerequisite to perform its triglyceride-hydrolyzing function in lipoproteins, and GPIHBP1 is exactly the very carrier responsible for translocating LPL to capillary lumen after binding it in the subendothelial space. Without the presence of GPIHBP1, LPL will be trapped in the intercelullar space, with no possibility to hydrolyze triglycerides in circulating chylomicrons and VLDLs $[28,29]$.

The human GPIHBP1 gene consists of 4 exons which encode a 184 amino acid protein. An acidic domain being able to bind LPL and chylomicrons is located in the N-terminus of GPIHBP1. Its C-terminus is encoded by exons 3 and 4, including a cysteine-rich lymphocyte antigen 6 (Ly6) motif as well as a carboxylterminal hydrophobic sequence involved in the addition of a GPI anchor [30]. A bunch of residues in the domain of C-terminus, such as Ser107, Thr124, and Leu135 also play a critical role in LPL binding and its transportation from subendothelial space to luminal capillary [31]. Therefore, deleting exons 3 and 4 will produce a crippled protein characterized by the absence of a domain indispensible for LPL binding and translocation as well as for anchoring LPL to the cell surface. This makes sense of the phenotype in 
cases with severe hyperlipidemia and also explains the lack of circulating LPL and LPL activity in heparin-processed plasma. Since the initial description of hyperlipidemia resulting from the variant of GPIHBP1 in 2007 [32], only about 50 cases have been reported worldwide [26, 33, 34]. Meanwhile, the genotypic spectrum based on HGMD has recorded 47 various pathogenic variants in GPIHBP1 that can result in severe hyperlipidemia, with most of them (33/47) identified as missense variants. Tendency of these variants towards any ethnicity or racial groups was not observed. Therefore, thorough analysis on these disease-causing variants probably linking to developmental malfunction and phenotypic changes is essential to pathogenesis clarifying and clinical treatment.

The patient in our study predominantly manifested fever, vomiting and convulsion, with unexpected discovery of severe hyperlipidemia and molecular genetic tests revealing the etiology. With the WES method, we found a novel homozygous variant, c.45_48dupGCGG(Pro17Alafs*22), in exons 1 of GPIHBP1, which, absent in all of the 100 healthy control samples, was inherited from both of her parents.

The c.45_48dupGCGG duplication variant in exon 1 may lead to the substitution of the remaining 168 Cterminal amino acid residues with 21 mutant ones, at a highly conserved position of this protein. Based on the clinical presentations, serum lipid level and bioinformatics study, an inference stood out from the potential pathogeneses that the disease of the proband was very likely to be attributed to the homozygous duplication variant, which, certainly, needs to be further verified by functional experiments in the future. Fortunately, these unexpected findings, together with timely and proper treatment, have resulted in a good prognosis observed in this patient. Furthermore, this orphan case gave us a lively lesson, and deepened our knowledge of this disease.

\section{Conclusions}

In conclusion, we presented a comprehensive delineation of the first Chinese infant patient with severe hyperlipidemia whose condition was rooted in a novel homozygous variant of the GPIHBP1 gene. In view of the occult and atypical clinical manifestation, we consider routine blood biochemical tests equally crucial to the diagnosis just as the molecular genetic detection is. As an integal part of the tools for a confirmed diagnosis, molecular genetic testing is strongly recommended in prenatal screening and postnatal diagnostic testing for those who are suspected or already identified as hereditary hyperlipidemia cases based on their clinical manifestation and serum lipid testing. Besides, for a proband with a diagnosis of hereditary hyperlipidemia or the carrier of relevant variants, the prenatal genetic testing, if available, should also be carried out in his or her family for future pregnancies.

\section{Abbreviations}

TG

triglyceride

TC

total cholesterol 
HDL-C

high-density lipoprotein cholesterol

LDL-C

low-density lipoprotein cholesterol

LPL

lipoprotein lipase

APOC2

apolipoprotein CII

APOA5

apolipoprotein AV

LMF1

lipase maturation factor 1

GPIHBP1

glycosylphosphatidylinositol-anchored highdensity lipoprotein-binding protein 1

Ly6

lymphocyte antigen 6

MCT

medium chain triglycerides

TPN

total parenteral nutrition

WES

whole exome sequencing

BWA

Burrows-Wheeler Aligner

GATK

Genome Analysis Toolkit

SNVs

single-nucleotide variants

OMIM

Online Mendelian Inheritance in Man

FATHMM

Functional Analysis through Hidden Markov Models

CVD

cardiovascular disease.

\section{Declarations}

\section{Ethics approval and consent to participate}

This study was approved by the Ethics Committee of Guangdong Women and Children Hospital. Before the study, the proband's parents had authorized the photographs, clinical examinations and laboratory 
tests, as well as publication of the research. Written informed consent was obtained from each participant. All the procedures conducted in our study were strictly in accordance with the Declaration of Helsinki.

\section{Consent for publication}

Written informed consent was given by the parents for the publication of all associated data and images.

\section{Availability of data and materials}

All data collected or generated for analysis in this study are included in this manuscript [and its Supplementary files].

\section{Competing interests}

The authors declare no any competing interests.

\section{Funding}

This research was funded by Natural Science Foundation of Guangdong Province (No. 2021A1515010969) and Research Project of Traditional Chinese Medicine Bureau of Guangdong Province (No. 20211046). The sponsors were not involved in study design, data analyzing or interpreting. Neither did they contribute to developing the report.

\section{Authors' contributions}

Xianhua Zheng and Ye Zhang made a great contribution to the study design, and also worked as two of the major participants in genetic analyses and manuscript drafting. Sisi Weiand Haimei OuYang were responsible for the patient's management and data collection. Jinqun Liangand Nuan Chen conducted the whole exome sequencing, variant verification and bioinformatics analysis of the variants. Weihong Zeng and Jianhui Jiang performed clinical routine biochemistry tests. Shu Liuand Zhiqing Wang conceived the study, at the same time worked as coordinators in this study, and assisted in manuscript drafting. All the authors have reviewed, provided comments and approved the manuscript as the final version.

\section{Acknowledgements}

We are indebted to all doctors, nurses and researchers for the follow-up assessment and data collection in the Children Inherited Metabolism and Endocrine Department, Guangdong Women and Children Hospital.

\section{Authors's contributions}

Xianhua Zheng and Ye Zhang made a great contribution to the study design, and also worked as two of the major participants in genetic analyses and manuscript drafting. Sisi Weiand Haimei OuYang were 
responsible for the patient's management and data collection. Jinqun Liangand Nuan Chen conducted the whole exome sequencing, variant verification and bioinformatics analysis of the variants. Weihong Zeng and Jianhui Jiang performed clinical routine biochemistry tests. Shu Liuand Zhiqing Wang conceived the study, at the same time worked as coordinators in this study, and assisted in manuscript drafting. All the authors have reviewed, provided comments and approved the manuscript as the final version.

\section{Author details}

${ }^{1}$ Children Inherited Metabolism and Endocrine Department, Guangdong Women and Children Hospital, No. 521 XingNan Road, Panyu District, Guangzhou, Guangdong 511400, P.R.China. ${ }^{2}$ Guangdong Provincial Key Laboratory of Gastroenterology, Department of Gastroenterology, Nanfang Hospital, Southern Medical University, No. 1838 North Guangzhou Avenue, Baiyun District, Guangzhou, Guangdong 510515, P.R.China. ${ }^{3}$ Department of Clinical Laboratory, Guangdong Women and Children Hospital, No. 521 XingNan Road, Panyu District, Guangzhou, Guangdong 511400, P.R.China.

\section{References}

1. Hutchison B, Collins J, Makar RS, Dzik W. Retrospective analysis of outcomes in patients with acute hypertriglyceridemic pancreatitis treated without therapeutic plasma exchange. Transfusion. 2021 Feb;61(2):537-45.

2. Brahm A, Hegele RA. Hypertriglyceridemia. Nutrients. 2013 Mar 22;5(3):981-1001.

3. Falko JM. Familial Chylomicronemia Syndrome: A Clinical Guide For Endocrinologists. Endocr Pract. 2018 Aug;24(8):756-63.

4. Gotoda T, Shirai K, Ohta T. Research Committee for Primary Hyperlipidemia, Research on Measures against Intractable Diseases by the Ministry of Health, Labour and Welfare in Japan. Diagnosis and management of type I and type V hyperlipoproteinemia. J Atheroscler Thromb. 2012;19(1):1-12.

5. Taghizadeh E, Ghayour-Mobarhan M, Ferns GA, Pasdar A. A novel variant in LPL gene is associated with familial combined hyperlipidemia. Biofactors. 2020 Jan;46(1):94-9.

6. Péterfy M, Bedoya C, Giacobbe C, Pagano C, Gentile M, Rubba P, Fortunato G, Di Taranto MD. Characterization of two novel pathogenic variants at compound heterozygous status in lipase maturation factor 1 gene causing severe hypertriglyceridemia. J Clin Lipidol. 2018 SepOct;12(5):1253-9.

7. Okubo M, Toromanovic A, Ebara T, Murase T. Apolipoprotein C-II Tuzla: a novel large deletion in APOC2 caused by Alu-Alu homologous recombination in an infant with apolipoprotein C-II deficiency. Clin Chim Acta. 2015 Jan 1;438:148-53.

8. Xenoulis PG, Tate NM, Bishop MA, Steiner JM, Suchodolski JS, Furrow E. Vet J. Sequence analysis of the coding regions of the apolipoprotein $\mathrm{C} 2(A P O C 2)$ gene in Miniature Schnauzers with idiopathic hypertriglyceridemia. 2020 Nov;265:105559. 
9. Ariza MJ, Rioja J, Ibarretxe D. Spanish Dyslipidemia Registry. Molecular basis of the familial chylomicronemia syndrome in patients from the National Dyslipidemia Registry of the Spanish Atherosclerosis Society. J Clin Lipidol. 2018 Nov-Dec;12(6):1482-92.e3.

10. Wójcik C, Fazio S, Mclntyre AD, Hegele RA. Co-occurrence of heterozygous CREB3L3 and APOA5 nonsense variants and polygenic risk in a patient with severe hypertriglyceridemia exacerbated by estrogen administration. J Clin Lipidol. 2018 Sep-Oct;12(5):1146-50.

11. Chen WW, Yang Q, Li XY, Shi XL, Pu N, Lu GT, et al. Identification of a novel and heterozygous LMF1 nonsense mutation in an acute pancreatitis patient with severe hypertriglyceridemia, severe obesity and heavy smoking. Lipids Health Dis. 2019 Mar;18(1):68. 18(.

12. Serveaux Dancer $M$, Di Filippo $M$, Marmontel $O$, Valéro R, Piombo Rivarola MDC, Peretti $N$, et al. New rare genetic variants of $L M F 1$ gene identified in severe hypertriglyceridemia. J Clin Lipidol. 2018 SepOct;12(5):1244-52.

13. Lima JG, Helena C, Nobrega L, Moura Bandeira FT, Pires Sousa AG, Medeiros de Araujo Macedo TB, Cavalcante Nogueira AC, et al. A novel GPIHBP1 mutation related to familial chylomicronemia syndrome: A series of cases. Atherosclerosis. 2021 Apr;322:31-8.

14. Paquette $M$, Hegele RA, Paré $G$, Baass A. A novel mutation in GPIHBP1 causes familial chylomicronemia syndrome. J Clin Lipidol. 2018 Mar-Apr;12(2):506-10.

15. loka RX, Kang MJ, Kamiyama S, Kim DH, Magoori K, Kamataki A, et al. Expression cloning and characterization of a novel glycosylphosphatidylinositol-anchored high density lipoprotein-binding protein, GPI-HBP1. J Biol Chem. 2003 Feb 28;278(9):7344-9.

16. Davydov EV, Goode DL, Sirota M, Cooper GM, Sidow A, Batzoglou S. Identifying a high fraction of the human genome to be under selective constraint using GERP++. PLoS Comput Biol. 2010;6:e1001025.

17. Richards S, Aziz N, Bale S, ACMG Laboratory Quality Assurance Committee. Standards and guidelines for the interpretation of sequence variants: a joint consensus recommendation of the American College of Medical Genetics and Genomics and the Association for Molecular Pathology. Genet Med. 2015;17:405-24.

18. Olivecrona G. Role of lipoprotein lipase in lipid metabolism. Curr Opin Lipidol. 2016 Jun;27(3):23341.

19. Gill PK, Dron JS, Dilliott AA, Mclntyre AD, Cao H, Wang J, et al. Ancestry-specific profiles of genetic determinants of severe hypertriglyceridemia. J Clin Lipidol. 2021 Jan-Feb;15(1):88-96.

20. Yang Q, Pu N, Li XY, Shi XL, Chen WW, Zhang GF, et al. Digenic Inheritance and Gene-Environment Interaction in a Patient With Hypertriglyceridemia and Acute Pancreatitis. Front Genet. $2021 \mathrm{Apr}$ 16;12:640859.

21. Dron JS, Hegele RA. Genetics of Hypertriglyceridemia. Front Endocrinol (Lausanne). 2020 Jul 24;11:455.

22. Beigneux AP, Davies BS, Gin P, Weinstein MM, Farber E, Qiao X, et al. Glycosylphosphatidylinositolanchored high-density lipoprotein-binding protein 1 plays a critical role in the lipolytic processing of 
chylomicrons. Cell Metab. 2007 Apr;5(4):279-91.

23. Meng X, Zeng W, Young SG, Fong LG. GPIHBP1, a partner protein for lipoprotein lipase, is expressed only in capillary endothelial cells. J Lipid Res. 2020 May;61(5):591.

24. Young SG, Fong LG, Beigneux AP, Allan CM, He C, Jiang H, et al. GPIHBP1 and Lipoprotein Lipase, Partners in Plasma Triglyceride Metabolism. Cell Metab. 2019 Jul 2;30(1):51-65.

25. Liu C, Li L, Guo D, Lv Y, Zheng X, Mo Z, et al. Lipoprotein lipase transporter GPIHBP1 and triglyceriderich lipoprotein metabolism. Clin Chim Acta. 2018 Dec;487:33-40.

26. Rabacchi C, D'Addato S, Palmisano S, Lucchi T, Bertolini S, Calandra S, et al. Clinical and genetic features of 3 patients with familial chylomicronemia due to mutations in GPIHBP1 gene. J Clin Lipidol. 2016 Jul-Aug;10(4):915-21.

27. Larsson M, Allan CM, Heizer PJ, Tu Y, Sandoval NP, Jung RS, et al. Impaired thermogenesis and sharp increases in plasma triglyceride levels in GPIHBP1-deficient mice during cold exposure. J Lipid Res. 2018 Apr;59(4):706-713.

28. Surendran RP, Udayyapan SD, Clemente-Postigo M, Havik SR, Schimmel AWM, Tinahones F, et al. Decreased GPIHBP1 protein levels in visceral adipose tissue partly underlie the hypertriglyceridemic phenotype in insulin resistance. PLoS One. 2018 Nov 8;13(11):e0205858.

29. Allan CM, Jung CJ, Larsson M, Heizer PJ, Tu Y, Sandoval NP, et al. Mutating a conserved cysteine in GPIHBP1 reduces amounts of GPIHBP1 in capillaries and abolishes LPL binding. J Lipid Res. 2017 Jul;58(7):1453-1461.

30. Birrane G, Beigneux AP, Dwyer B, Strack-Logue B, Kristensen KK, Francone OL, et al. Structure of the lipoprotein lipase-GPIHBP1 complex that mediates plasma triglyceride hydrolysis. Proc Natl Acad Sci U S A. 2019 Jan;29(5):1723-32. 116(.

31. Beigneux AP, Davies BS, Tat S, Chen J, Gin P, Voss CV, et al. Assessing the role of the glycosylphosphatidylinositol-anchored high density lipoprotein-binding protein 1 (GPIHBP1) threefinger domain in binding lipoprotein lipase. J Biol Chem. 2011 Jun 3;286(22):19735-43. doi: 10.1074/jbc.M111.242024.

32. Wang J, Hegele RA. Homozygous missense mutation (G56R) in glycosylphosphatidylinositolanchored high-density lipoprotein-binding protein 1 (GPI-HBP1) in two siblings with fasting chylomicronemia (MIM 144650). Lipids Health Dis. 2007 Sep;20:6:23.

33. Hegele RA, Berberich AJ, Ban MR, Wang J, Digenio A, Alexander VJ, et al. Clinical and biochemical features of different molecular etiologies of familial chylomicronemia. J Clin Lipidol. 2018 JulAug;12(4):920-7.e4.

34. Ariza MJ, Martínez-Hernández PL, Ibarretxe D, Rabacchi C, Rioja J, Grande-Aragón C, et al. Novel mutations in the GPIHBP1 gene identified in 2 patients with recurrent acute pancreatitis. J Clin Lipidol. 2016 Jan-Feb;10(1):92-100.e1.

\section{Figures}




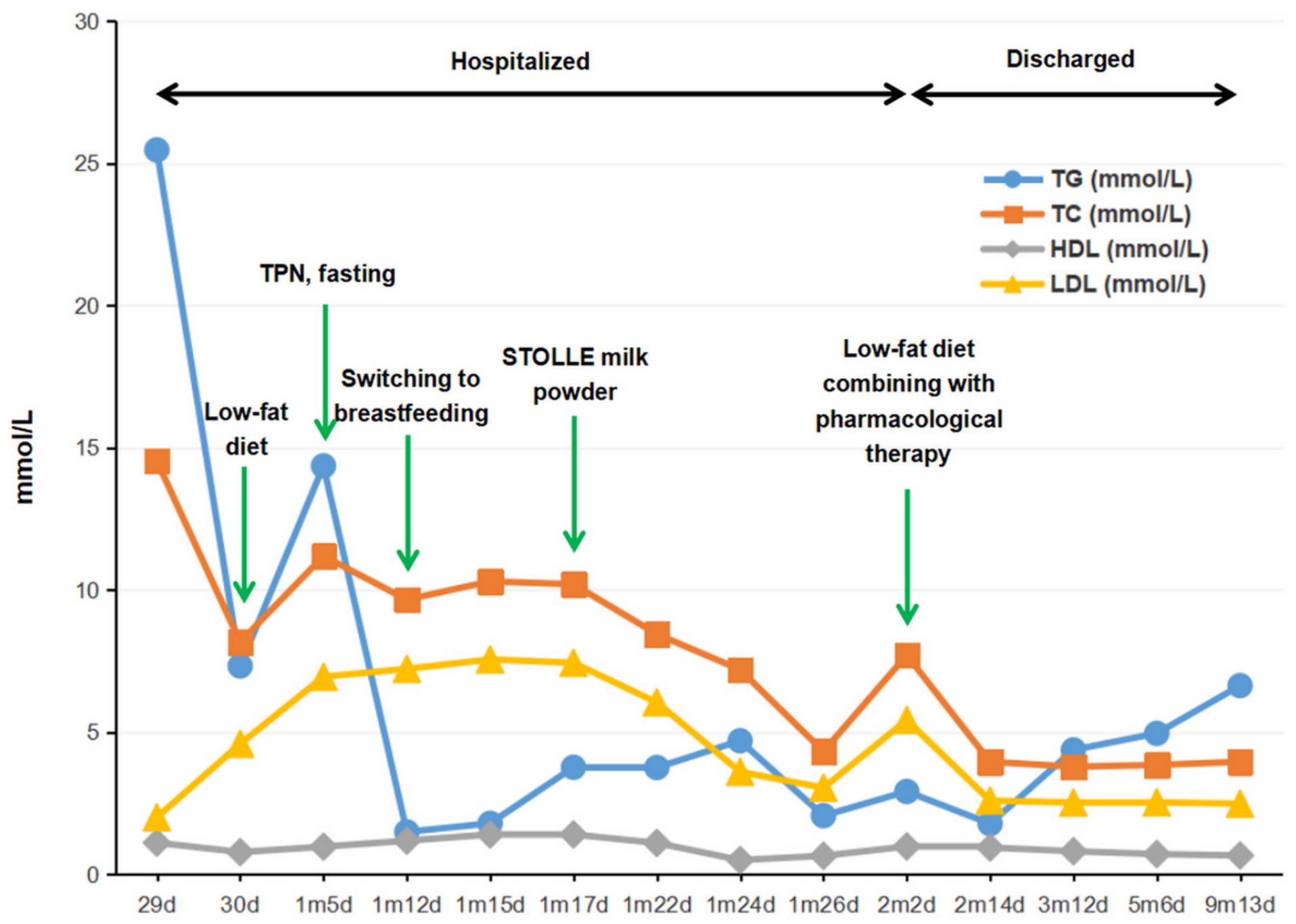

Figure 1

Flow-chart illustrating the management procedure and the serum levels of TG, TC, HDL-C and LDL-C of the proband. TG, triglyceride; TC, total cholesterol; HDL-C, high-density lipoprotein cholesterol; LDL-C, lowdensity lipoprotein cholesterol; TPN, total parenteral nutrition. 


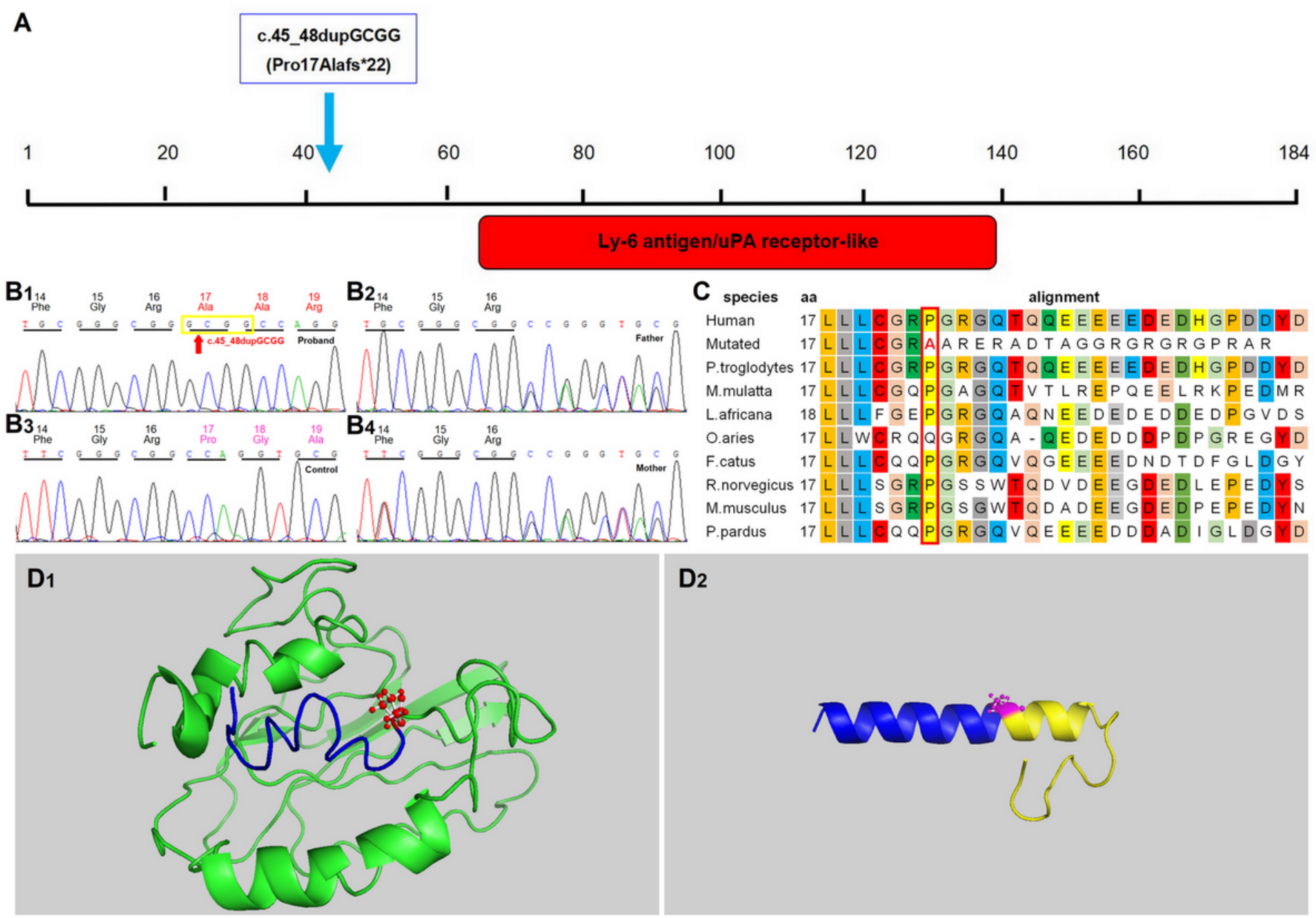

\section{Figure 2}

Identification of the variant in GPIHBP1 gene. (A) Graphical view of the protein domain and structure of GPIHBP1. The red box stands for the Ly- 6 antigen/uPA receptor-like domain (IPR016054). The homozygous variant c.45_48dupGCGG(p.Pro17Alafs*22) is marked by wathet blue arrow. (B) Sequence chromatograms of the GPIHBP1 variant. B1-B4 indicates the variant sequences of c.45_48dupGCGG in the proband, his father, mother and normal controls, respectively. The duplication variant site is indicated by red arrow. (C) BLAST comparison of the sequence around amino acids 17 in orthologs of GPIHBP1 among various species. The block and colored characters represent highly conserved amino acids among these species, while the white shading stands for inconsistent residues. (D) Structural difference between wild-type and C-terminal frameshift GPIHBP1 proteins. Efforts were made to predict the functions of the mutant protein and the wild-type (full-length) GPIHBP1. The full length wild-type protein is exhibited in the left panel (D1), and the mutant protein (p.Pro17Alafs ${ }^{\star 22}$ ) in the right panel (D2). As shown in the mutant GPIHBP1 proteins (D2) model, the frameshift originated from p.Pro17Alafs $* 22$ variant is highlighted in yellow, corresponding to the wild-type portion depicted in green (D1). Meanwhile, the consistent part of the $\mathrm{N}$-terminus of the wild-type and the mutant protein is show in blue. It can be seen that the variant compromises the original structure and integrity of the GPIHBP1 protein. 


\section{Supplementary Files}

This is a list of supplementary files associated with this preprint. Click to download.

- Supplementarydocumentgeneticanalysis.doc

- Supplementarytable1.doc

- Supplementarytable2.doc 